\title{
Seroprevalence of Borrelia IgM and IgG Antibodies in Healthy Individuals: A Caution Against Serology Misinterpretations and Unnecessary Antibiotic Treatments
}

\author{
Zuzana Strizova, Daniel Smrz, and Jirina Bartunkova
}

\begin{abstract}
In Lyme disease, the interpretation of diagnostic assays is often misunderstood. Cross-reactions of Borrelia proteins with antigens from other bacterial species are well known. Therefore, to diagnose Lyme disease, the finding of positive IgM antibodies must be accompanied by objectively verified clinical signs and a history of a possible tick exposure. Positive Borrelia $\operatorname{IgM}$ antibodies in healthy individuals with nonspecific clinical symptoms are likely a false-positive result for Lyme disease and neither long-term antibiotic treatment nor cycling of different antibiotic regimens is beneficial. To date, there is clear evidence that positive serology does not indicate infection with Borrelia species. Borrelia serology has been reported to be positive for months or years in $\sim 20 \%$ of healthy patients who had experienced Lyme disease in the past. Thus, serology as a single diagnostic tool has a very limited value and should be used only to support clinically suspected cases.
\end{abstract}

Keywords: Borrelia, IgM antibodies, serology, diagnosis, Lyme disease

$\mathbf{L}$ YME DISEASE IS A COMMON vector-borne disease caused by spirochetal bacteria from the genus Borrelia, where the most prevalent pathogens in humans are Borrelia burgdorferi sensu stricto, Borrelia afzelii, and Borrelia garini (Wilson et al. 2012, Sanchez 2015). The disease is usually spread by a tick bite, causing a common sign known as erythema migrans (Wilson et al. 2012, Sanchez 2015). The early stages of Lyme disease are easily treated by antibiotics (Sanchez 2015). However, a long-term antibiotic treatment could be damaging for patients with persisting IgM antibodies (Seriburi et al. 2012, Nemeth et al. 2016).

During the past few years, several principles regarding diagnostic assays have evolved but, still, the diagnosis of Lyme disease is often misunderstood. The diagnosis should be established clinically based on symptoms, objective clinical findings, and a history of possible exposure to infected tick (Marques 2015, Nemeth et al. 2016). Typical erythema migrans does not require serologic confirmation (Dessau et al. 2018). Serology may assist in the decisionmaking process but cannot determine the diagnosis as a single diagnostic tool. To date, there is clear evidence that positive serology itself does not indicate infection with
Borrelia species (Gregson et al. 2015, Marques 2015, Nemeth et al. 2016). As a single diagnostic tool, serology has only a limited value, however, can still be used to support clinically suspected cases (Gregson et al. 2015, Marques 2015, Nemeth et al. 2016, Dessau et al. 2018).

In humans infected with $B$. burgdorferi, the serology can be negative in the early stage of the disease as the body may not have produced a detectable quantity of specific antibodies (Gregson et al. 2015). Nevertheless, Borrelia serology has been reported to be positive for months or even years in $~ 20 \%$ of healthy patients who had experienced Lyme disease in the past (Gregson et al. 2015). A high prevalence of B. burgdorferi antibodies in healthy individuals is evident among different geographical areas and different subgroups of individuals (Dessau et al. 2018) (Fig. 1). The phenomenon of persisting IgM response does not indicate a persistent infection with $B$. burgdorferi (Leeflang et al. 2016, Dessau et al. 2018) and shall not lead to repetitive antibiotic treatment. It also appears that in early stages of Lyme disease, IgM serology detection devaluates specificity of other diagnostic testing (Leeflang et al. 2016).

Treatment of Lyme disease should never be based on the presence of positive IgG alone. This applies to IgM

Department of Immunology, Second Faculty of Medicine, Charles University in Prague and University Hospital Motol, Prague, Czech Republic. 


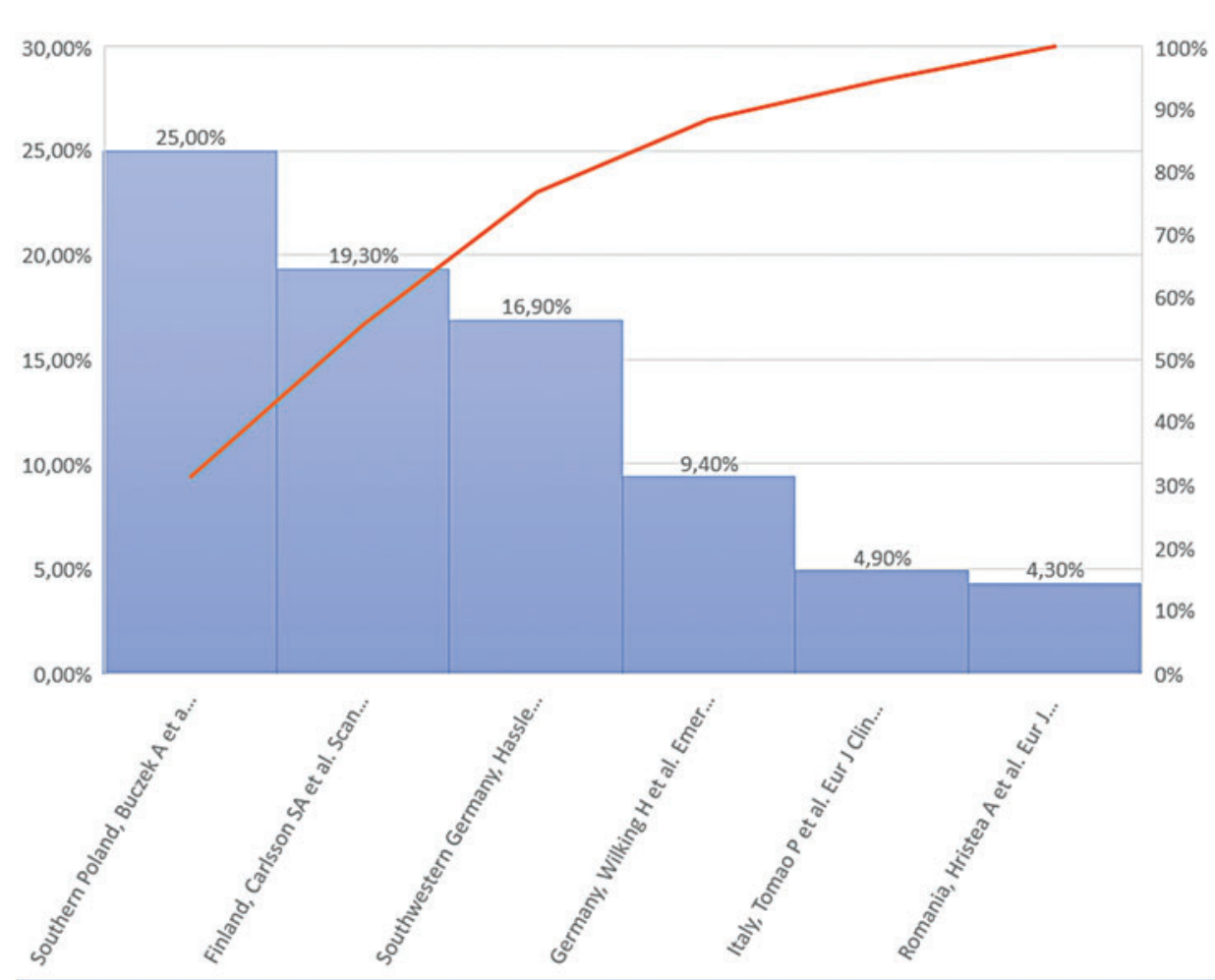

\section{Seroprevalence of \\ Borrelia burgdorferi \\ antibodies among \\ selected geographical \\ areas and specific \\ subpopulations}

FIG. 1. Seroprevalence of Borrelia burgdorferi antibodies among selected geographical areas and specific subgroups of individuals. Color images are available online.

antibodies as well, as the cross-reactions of Borrelia proteins with antigens from other bacterial species are well known (Ryffel et al. 1998). The Lyme disease IgM testing is valuable in the diagnosis of the early stage Lyme disease only if the finding is accompanied by objectively verified clinical signs and a history of a tick bite or a possible tick exposure (Seriburi et al. 2012, Dessau et al. 2018). Positive IgM in patients with nonspecific clinical symptoms is likely a false-positive result for Lyme disease, and neither long-term antibiotic treatment nor cycling of different antibiotic regimens is beneficial (Nemeth et al. 2016). To repeat serological testing after treatment and evaluate the IgM and IgG dynamics are strongly discouraged due to the individual variability of immune responses. Serology alone does not reflect the suc- cess/failure of the antibiotic treatment, nor the clinical course of the disease (Leeflang et al. 2016, Dessau et al. 2018).

The use of Western blot and enzyme-linked immunosorbent assay (ELISA) in the diagnosis of Lyme disease remains controversial (Hsieh et al. 2007). The use of immunoblot alone is not recommended and its combination with ELISA causes difficulties in optimization and standardization (Leeflang et al. 2016, Dessau et al. 2018). It has already been reported that some individuals remain immunoblot-positive for years after the infection (Leeflang et al. 2016). The specificity of detecting antibodies in erythema migrans patients with ELISA/immunoblot was reported close to $90 \%$, whereas the sensitivity ranged from $40 \%$ to $61 \%$ (Leeflang et al. 2016). The sensitivity of serology

\begin{tabular}{|c|c|c|c|}
\hline \multicolumn{2}{|c|}{ Sensitivity of serological testing (IgM and IgG) } & Clinically suspected case & \\
\hline \multirow[t]{7}{*}{$0 \%$} & $>99 \%$ & Lyme carditis & $>80 \%$ \\
\hline & & Lyme arthritis & $>90 \%$ \\
\hline & & Erythema migrans & $>40 \%$ \\
\hline & & Neuroborreliosis - late manifestation & $>99 \%$ \\
\hline & & Neuroborreliosis - early manifestation & $>60 \%$ \\
\hline & & Ocular manifestations & \\
\hline & & Lymphocytoma & $>80 \%$ \\
\hline Data not available & & Acrodermatitis chronica atrophicans & $>90 \%$ \\
\hline
\end{tabular}

FIG. 2. The sensitivities of serological tests differ among suspected clinical cases. Color images are available online. 
testing is linked to the clinical manifestation of Lyme disease and varies individually (Fig. 2). Therefore, the serologic data must be interpreted and managed wisely. According to recent findings, two-tiered tests do not provide superior benefit as compared with single diagnostic test (Leeflang et al. 2016, Dessau et al. 2018). However, highly sensitive screening tests including VlsE antigen may, together with positive $\mathrm{IgG}$, confirm a suspected case of Lyme disease (Leeflang et al. 2016, Dessau et al. 2018).

PCR tests for Borrelia DNA can also be performed (Hsieh et al. 2007). However, positive results can only occur in tissues with a high accumulation of bacteria at the site of infection. Since Lyme disease is a systemic condition, PCR is not widely performed for the diagnosis of Lyme disease. However, it may be used for the diagnosis of Lyme arthritis and, therefore, represents a highly sensitive way of detecting ospA DNA in synovial fluid (Hsieh et al. 2007).

To avoid repeating unnecessary antibiotic regimens, it is crucial to apprehend the recent guidelines for the diagnosis of Lyme disease (Leeflang et al. 2016, Dessau et al. 2018).

\section{Author Disclosure Statement}

The authors Z.S. and D.S. declare that they have no conflict of interest regarding the publication of this article. J.B. is a part-time employee and a minority shareholder of SOTIO, a.s., a company developing cell-based immunotherapy. The article has been approved for publication by all authors and is not currently under review or submitted elsewhere.

\section{Funding Information}

No funding was received for this article.

\section{References}

Dessau RB, van Dam AP, Fingerle V, Gray J, et al. To test or not to test? Laboratory support for the diagnosis of Lyme borreliosis-Author's reply. Clin Microbiol Infect 2018; 24: 211-212.
Gregson D, Evans G, Patrick D, Bowie W. Lyme disease: How reliable are serologic results? CMAJ 2015; 187:1193-1194.

Hsieh YF, Liu HW, Hsu TC, Wei JC, et al. Serum reactivity against Borrelia burgdorferi OspA in patients with rheumatoid arthritis. Clin Vaccine Immunol 2007; 14:1437-1441.

Leeflang MM, Ang CW, Berkhout J, et al. The diagnostic accuracy of serological tests for Lyme borreliosis in Europe: A systematic review and meta-analysis. BMC Infect Dis 2016; 16:140.

Marques AR. Laboratory diagnosis of Lyme disease: Advances and challenges. Infect Dis Clin North Am 2015; 29:295-307.

Nemeth J, Bernasconi E, Heininger U, Abbas M, et al. Update of the Swiss guidelines on post-treatment Lyme disease syndrome. Swiss Med Wkly 2016; 146:w14353.

Rose CD, Fawcett PT, Singsen BH, Dubbs SB, et al. Use of western blot and enzyme-linked immunosorbent assays to assist in the diagnosis of Lyme disease. Pediatrics 1991; 88:465-470.

Ryffel K, Peter O, Binet L, Dayer E. Interpretation of immunoblots for Lyme borreliosis using a semiquantitative approach. Clin Microbiol Infect 1998; 4:205-212.

Sanchez JL. Clinical manifestations and treatment of Lyme disease. Clin Lab Med 2015; 35:765-778.

Seriburi V, Ndukwe N, Chang Z, Cox ME, et al. High frequency of false positive IgM immunoblots for Borrelia burgdorferi in clinical practice. Clin Microbiol Infect 2012; 18:1236-1240.

Wilson TC, Legler A, Madison KC, Fairley JA, et al. Erythema migrans: A spectrum of histopathologic changes. Am J Dermatopathol 2012; 34:834-837.

Address correspondence to: Zuzana Strizova

Department of Immunology

Second Faculty of Medicine

Charles University in Prague and University Hospital Motol

V Uvalu 84

Prague 15006

Czech Republic

E-mail: zuzana.strizova@fnmotol.cz 